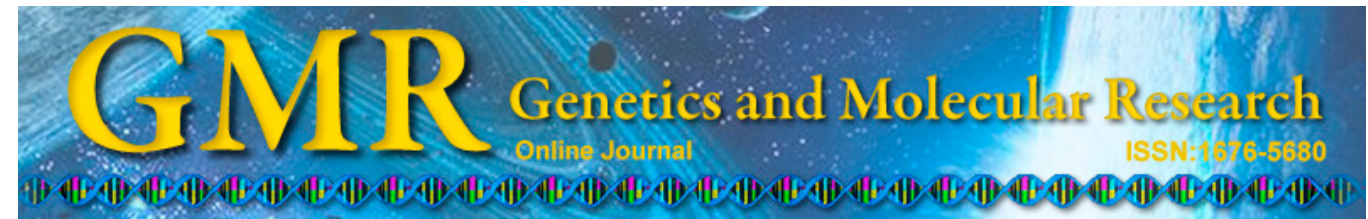

\title{
Molecular characterization of a cytosolic manganese superoxide dismutase from the Chinese mitten crab, Eriocheir sinensis
}

\author{
D.X. Zhao ${ }^{1,2}$, L.Q. Chen ${ }^{1}$, J.G. Qin ${ }^{3}$, C.J. Qin ${ }^{1}$, H. Zhang ${ }^{1}$, P. Wu ${ }^{1}$ and \\ E.C. $\mathbf{L i}^{1}$ \\ ${ }^{1}$ Department of Biology, East China Normal University, Shanghai, China \\ ${ }^{2}$ School of Life Science and Food Engineering, Nanchang University, \\ Jiangxi, China \\ ${ }^{3}$ School of Biological Sciences, Flinders University, Adelaide, Australia \\ Corresponding author: L.Q. Chen \\ E-mail: lqchen@bio.ecnu.edu.cn
}

Genet. Mol. Res. 13 (4): 9429-9442 (2014)

Received September 6, 2013

Accepted August 13, 2014

Published November 11, 2014

DOI http://dx.doi.org/10.4238/2014.November.11.8

\begin{abstract}
A cytosolic manganese superoxide dismutase gene (Es$c M n S O D$ ) was cloned from the Chinese mitten crab Eriocheir sinensis, using reverse transcription-polymerase chain reaction and the rapid amplification of cDNA ends. The open reading frame of Es-cMnSOD is $867 \mathrm{bp}$ in length and encodes a 288 -amino acid protein without a signal peptide. The calculated molecular mass of the translated protein of Es-cMnSOD is $31.43 \mathrm{kDa}$, with an estimated isoelectric point of 6.30. The deduced amino acid sequence of $E s-c M n S O D$ has similarities of $90,89,84,87$, and $81 \%$ to those of white shrimp Litopenaeus vannamei MnSOD, black tiger shrimp Penaeus monodon MnSOD, giant freshwater prawn Macrobrachium rosenbergii MnSOD, blue crab Callinectes sapidus MnSOD, and red swamp crayfish Procambarus clarkii MnSOD, respectively. Es-cMnSOD contains a manganese superoxide dismutase domain (DVWEHAYY) and 4 conserved amino acids responsible for binding manganese. Es-cMnSOD was expressed
\end{abstract}


in the hemocytes, eyestalk, muscle, intestine, gill, and hepatopancreas. Es-cMnSOD transcripts in hemocytes of E. sinensis increased at 1.5 and $48 \mathrm{~h}$ after injection of Aeromonas hydrophila, indicating that the induction of the SOD system response occurred within a short period of time. This study suggests that MnSOD may play a critical role in crab immunity, allowing efficient activation of an early innate immune response in the crab.

Key words: Cytosolic manganese superoxide dismutase; Hemocyte; Eriocheir sinensis; Aeromonas hydrophila

\section{INTRODUCTION}

Phagocytosis is an important mechanism for immune defense in crustacean via engulfing bacteria in macrophage when hemocytes become infected with bacteria (Lee and Söderhäll, 2002; Smith et al., 2003). At this time, reactive oxygen species (ROS), including superoxide anion and hydrogen peroxide, are produced to kill the invading bacteria. However, when the ROS level exceeds the threshold level of the antioxidant system, cellular redoxhomeostasis is disturbed, thus leading to oxidative stress (Trachootham et al., 2008). ROS are widely recognized as dual-role players since they act as key mediators in a variety of cellular processes, and an elevated ROS level can have deleterious effects to cellular macromolecules (Muñoz et al., 2000; Johnson, 2002). To defend against oxidative damage, cells are equipped with myriad antioxidant enzymes to scavenge and detoxify the accumulated oxyradicals (Vaughan, 1997; Park et al., 2004; Arenas-Ríos et al., 2007).

Among the antioxidant enzymes, the superoxide dismutases (SODs) comprise the first defense system that catalyzes the dismutation of superoxide radicals to hydrogen peroxide and oxygen (McCord and Fridovich, 1969). Since the SODs were discovered in the 1960s (McCord and Fridovich, 1969), many types of SODs have been reported as metalloproteins in the cells of a wide range of organisms, including cytosolic $\mathrm{Cu} / \mathrm{ZnSOD}, \mathrm{MnSOD}$, FeSOD and NiSOD (Bannister et al., 1987; Fridovich, 1995).

MnSOD containing manganese ions was primarily found in the mitochondrial matrix (mMnSOD) (Weisiger and Fridovich, 1973), but some cytosolic MnSODs (cMnSOD) have also been reported in crabs, lobsters, prawns, and shrimp (Brouwer et al., 2003). The former was found in bacteria, plants, invertebrates, and vertebrates, whereas the latter was found only in crustaceans (Lin et al., 2010). Recently, MnSOD has received much attention because it is a major scavenger of damaging ROS metabolites in the mitochondrial matrix ( $\mathrm{Li}$ et al., 2011). More importantly, the SOD protein has been also demonstrated to play an important role in cellular differentiation and tumorigenesis (St Clair et al., 1994) in the immune response induced by bacteria (Jung et al., 2005; Cheng et al., 2006), viruses (Zhang et al., 2007), or exposure toxic chemical (Kim et al., 2007).

To date, many MnSOD orthologs have been characterized in mammalian (Ho et al., 1991; Wan et al., 1994; Meyrick and Magnuson, 1994; Jones et al., 1995), fish (Lin et al., 2009), and invertebrate (Gómez-Anduro et al., 2006; Bao et al., 2008; Zhang et al., 2011; Yu et al., 2011) species. In decapod crustaceans, some MnSODs have also been cloned, including the pacific white shrimp Litopenaeus vannamei (DQ005531; DQ029053), tiger shrimp 
Penaeus monodon (AY726542), Chinese shrimp Fenneropenaeus chinensis (GQ168792), kuruma shrimp Marsupenaeus japonicus (GQ181123), freshwater prawn Macrobrachium rosenbergii (EU077526), grass shrimp Palaemontes pugio [AY211084, partial complementary DNA (cDNA)], giant mud crab Scylla serrata (GU213434), blue crab Callinectes sapidus (AF264030), and hydrothermal crab Bythograea thermydron (FM242567). Recently, the characterization of cMnSOD and its role of immunomodulation in $M$. rosenbergii (Cheng et al., 2006), L. vannamei (Gómez-Anduro et al., 2006), M. japonicas (Lin et al., 2010), and B. thermydron (Marchand et al., 2009) have also been reported.

In the last few decades, the cultured freshwater Chinese mitten crab Eriocheir sinensis has been an important economical species resource. Farming of this species has been a challenge due to opportunistic pathogens such as bacteria, viruses and rickettsia-like organisms (Wang and Gu, 2002; Wang et al., 2002; Xu et al., 2002) in deteriorated environments. Therefore, investigating the mechanisms of immune defense against pathogens in crabs may be beneficial to the management of crustacean-farming practices.

We previously identified an expressed sequence tag (EST) from E. sinensis cDNA library with sequence similarity to the freshwater prawn $M$. rosenbergii SOD (Zhao et al., 2009). Given the importance of SOD in immunity and the limited amount of molecular data available for $E$. sinensis, we further studied the SOD identified from this species in our previous EST study. The present study aimed at cloning and characterizing the E. sinensis SOD (designated as $E s-S O D$ ) by comparing its sequence to other known SODs, examining the expression levels of SOD in various tissues, and evaluating SOD expression in the hemocytes of crabs challenged with Aeromonas hydrophila. These results will provide a better understanding of the mechanisms underlying disease resistance and the process coping mechanisms with regard to oxidative stress in this species.

\section{MATERIAL AND METHODS}

\section{Animals}

Healthy crabs averaging $100 \mathrm{~g}$ (wet weight) were collected from a commercial crab farm near Shanghai, China, and acclimated at $22^{\circ} \pm 2^{\circ} \mathrm{C}$ for 1 week prior to being used for experimentation.

\section{RNA isolation from hemocytes and reverse transcription (RT)}

Hemolymph sampling and collection of hemocytes were conducted according to previously reported methods (Zhao et al., 2009). Total RNA was isolated from the hemocyte pellet and further purified using the Unizol Reagent Kit (Biostar, Shanghai, China) according to the manufacturer protocol. The total concentration of RNA was estimated by measuring the absorbance at $260 \mathrm{~nm}$. Total RNA ( $5 \mu \mathrm{g})$ was reversely transcribed using the MMLV reverse transcriptase (TaKaRa, Dalian, China) with the adapter primer (5'-GGCCACGCGTCGACT AGTACTTTTTTTTTTTTTTTTT-3') for 3'-rapid amplification of cDNA ends (RACE) and the 5'-CDS primer A [(5'-(T) $25 \mathrm{VN}-3^{\prime}, \mathrm{N}=\mathrm{A}, \mathrm{C}, \mathrm{G}$, or T; V = A, G, or C] for 5'-RACE to obtain the first-strand cDNA. Reaction conditions followed the recommendations of the kit manufacturer. 


\section{Cloning the full-length cDNA of SOD}

The full-length SOD cDNA of E. sinensis was obtained by the RT-polymerase chain reaction (RT-PCR) and the RACE methods. Two gene-specific primers, P1 (GSP1: 5'-CCGCC TCTAGCCTCCACTTGC-3') and P2 (GSP2: 5'-TTCTTGGAGCAGTAGCCGAGCC-3') were designed based on the sequence of hem_0002_D01.ab1 (Zhao et al., 2009). The 5'-region of the ES-SOD transcript was obtained via the 5'-RACE reaction using the SMART RACE cDNA Amplification Kit (TaKaRa) according to manufacturer instructions. The primers consisted of GSP1 with the universal primer A mix (5'-CTAATACGACTCACTATAGGGCAAGCAG TGGTATCAACGCAGAGT-3') for RACE to derive the 5'-untranslated region (UTR) and the signal peptide sequences. For 3'-RACE, the primers of GSP2 with the abridged universal amplification primer (5'-GGCCACGCGTCGACTAGTAC-3') were used for amplification of the target cDNA. The PCR fragments were subjected to electrophoresis on $1.0 \%$ agarose gel to determine differences in length. The amplified cDNA fragments were cloned into the pMD18-T vector $(\mathrm{TaKaRa})$ following manufacturer instructions. Recombinant bacteria were identified by blue/white screening and confirmed by PCR. Plasmids containing the inserted GST fragment were used as a template for DNA sequencing.

\section{Sequence analysis}

The homology searches of nucleotide and protein sequences were conducted with the BLAST algorithm at the National Center for Biotechnology Information (NCBI). The deduced amino acid sequence was analyzed with the Expert Protein Analysis System (http://www.expasy.org/). The SignalP 3.0 program was used to predict the presence and location of the signal peptide and the cleavage sites in amino acid sequences.

\section{Multiple-sequence alignment and phylogenetic analysis}

The full-length multiple alignment of the $E s-S O D$ sequence was compared to that of other SODs. Amino acid sequences from various species of arthropods were retrieved from the NCBI GenBank database and analyzed using the ClustalW Multiple Alignment program and the Multiple Align Show program. A neighbor-joining (N-J) phylogenetic tree was constructed using the MEGA software version 3.1 (Kumar et al., 2004). The reliability of the branching was tested using bootstrap re-sampling with 1000 pseudo-replicates.

\section{Expression of the ES-SOD gene in tissues}

The expression of Es-SOD messenger RNA (mRNA) in tissues was demonstrated by quantitative real-time RT-PCR (qRT-PCR) analysis. The hemocytes, hepatopancreas, muscles, gills, stomachs, and intestines were removed from the crabs. The tissues were blotted and weighed; they were then homogenized using the Unizol reagent (Biostar). Total RNA was extracted as described above. The first-strand cDNA was synthesized using the MMLV reverse transcriptase with $5 \mu \mathrm{g}$ total RNA. The genespecific primer pairs of Es-SOD QF (5'-CCCAGTTGATGACGTTGAAG-3') and Es- 
SOD QR (5'-CTCGGCTACTGCTCCAAGAA-3') were used to amplify the SOD transcript. The primers, $\beta$-actin F (5'-GCATCCACGAGACCACTTACA-3') and $\beta$-actin R (5'-CTCCTGCTTGCTGATCCACATC-3') were used to amplify the $\beta$-actin fragment that was used as the internal control.

\section{SOD expression in hemocytes after $A$. hydrophila challenge}

A total of 72 crabs were challenged. The crabs were randomly divided into 2 groups, and each treatment was applied in triplicate. Each crab in the challenge group received an injection of $100 \mu \mathrm{L}$ live $A$. hydrophila suspended in saline $\left(7.2 \times 10^{8} \mathrm{CFU} / \mathrm{mL}\right)$, while the crabs in the control group received the same volume of saline only. After treatment, the crabs were returned to the tanks, and 2 individuals from each group were randomly sampled at 0 , $1.5,3,6,12,24$, and $48 \mathrm{~h}$ post-injection. The qRT-PCR assay was performed using the SYBR Premix Ex Taq (TaKaRa) on an ABI StepOne real-time PCR system (Applied Biosystems, USA). Gene-specific primers of the Es-SOD QF and the Es-SOD QR with cDNA that had been reversely transcribed were used for the reaction according to manufacturer instructions. The primers $\beta$-actin $F$ and $\beta$-actin $R$ were used to amplify the $\beta$-actin fragment that was used as the internal control.

\section{Data analysis for SYBR Green-based qRT-PCR}

On a 96-well plate, each sample was run in triplicate along with the internal control gene. A dissociation curve analysis of amplification products was performed at the end of each PCR to confirm that only one PCR product was amplified and detected. After the PCR run, data analysis was performed with the StepOne Software version 2.0 (Applied Biosystems). Briefly, the threshold PCR cycle $(\mathrm{Ct})$ was defined as the cycle number at which a statistically significant increase in fluorescence of SYBR Green against the internal passive dye ROX $(\triangle \mathrm{Rn})$ was first detected. The copy number of the target gene and $\mathrm{Ct}$ values are inversely related; thus, a sample containing a higher number of copies of the target gene would have a lower $\mathrm{Ct}$ value than that of the sample with a lower number of copies of the same target gene. The differences in the $\mathrm{Ct}$ values of SOD and the corresponding internal control $\beta$-actin, (i.e., $\Delta \mathrm{Ct}$ ), were calculated to normalize any differences in the amount of total RNA added to the cDNA reaction mixture and the efficiency of RT reaction. The $\Delta \mathrm{Ct}$ for the control sample was subtracted from the $\Delta \mathrm{Ct}$ of the challenged sample. The difference was expressed as a $\Delta \Delta \mathrm{Ct}$ value that allowed measurement of the change in SOD expression in the challenged crabs relative to the control crabs. The expression level of ES-SOD, calculated by the formula $2^{-\Delta \Delta \mathrm{Ct}}$, was represented as an $\mathrm{n}$-fold difference relative to the control. The relative expression values were exported into a Microsoft Excel sheet for subsequent data analyses.

\section{Statistical analysis}

A multiple-comparison Duncan test was used to compare significant differences in $S O D$ gene expression between the control and challenged samples using the SPSS15.0 software. A significance level of $\mathrm{P}=0.05$ was chosen. 


\section{RESULTS}

\section{Nucleotide sequence of SOD cDNA and the deduced amino acid sequence}

A singleton sequence with significant similarity to the grant freshwater shrimp M. rosenbergii SOD gene was found from the crab hemocyte EST library. This partial sequence provided crucial DNA sequence information required for the 3'-RACE cloning and 5'-amplification of the complete $S O D$ of the crab. A 426-bp product of the 3'-end of $S O D$ was obtained by 3'-RACE. Approximately, $295 \mathrm{bp}$ of the 5'-end of $S O D$ were obtained by the RACE method. Finally, the full-length $S O D$ cDNA fragment of $E$. sinensis was obtained by overlapping $3 \mathrm{cDNA}$ fragment sequences. The nucleotide sequence and the deduced amino acid sequence are shown in Figure 1. The full-length of Es-SOD cDNA comprised of $1339 \mathrm{bp}$, containing $20 \mathrm{bp}$ in the $5^{\prime}-\mathrm{UTR}, 867 \mathrm{bp}$ in the open reading frame (ORF), 452 bp in 3'-UTR with a poly(A) tail of $25 \mathrm{bp}$, and a putative polyadenylation consensus signal (aataaa) of 19 nucleotides upstream of the poly(A) tail. The ORF encode a polypeptide of 288 amino acids without a signal peptide. The calculated molecular mass of the mature protein was $31.43 \mathrm{kDa}$, with an estimated isoelectric point of 6.30 . The Es-SOD cDNA sequence and its deduced amino acid sequence were submitted to the NCBI GenBank under accession No. FJ617306.

\section{Comparison of SOD sequences and motifs of $E$. sinensis with other species}

Sequence comparisons of the Es-SOD deduced amino acids showed 90, 89, 84, 87 , and $81 \%$ similarities to white shrimp L. vannamei MnSOD, P. monodon MnSOD, $M$. rosenbergii MnSOD, C. sapidus MnSOD, and Procambarus clarkii MnSOD, respectively. Multiple alignment of $E S-S O D$ with the SOD of different crustaceans indicated that they share some conserved motifs, including a general MnSOD domain from the 172nd to the 279th amino acid, a putative cMnSOD signature sequence from the 244-251th amino acids (DVWEHAYY), and 4 putative manganese binding sites (i.e., H111, H159, D244, and H248) (Figure 2). The high homology of the Es-SOD sequence to the MnSOD of other decapod species indicates that the Es-SOD cloned in E. sinensis belonged to the MnSOD family (designated as $E s-M n S O D$ ) and might play a similar role as that of other MnSOD in response to similar stresses in other species.

\section{Phylogenetic analysis}

To examine the molecular phylogeny of $E s-M n S O D, 53 \mathrm{MnSOD}$ orthologs from different taxa of arthropods were aligned and a phylogenetic tree was constructed using the N-J method (Table 1). The mitochondrial and cytosolic branches split and formed 2 major groups. All of the mMnSODs were clustered together into a subgroup, and the cMnSODs were clustered into another group, which was consistent with the belief of a common origin for these 2 SODs with different subcellular compartmentalizations. The $E s-M n S O D$ was placed within the cMnSOD monophyletic clade, close to its ortholog from Cardisoma armatum (Figure 3), indicating that the SOD obtained from E. sinensis should be classified as a cMnSOD (designated as $E S-c M n S O D)$. 


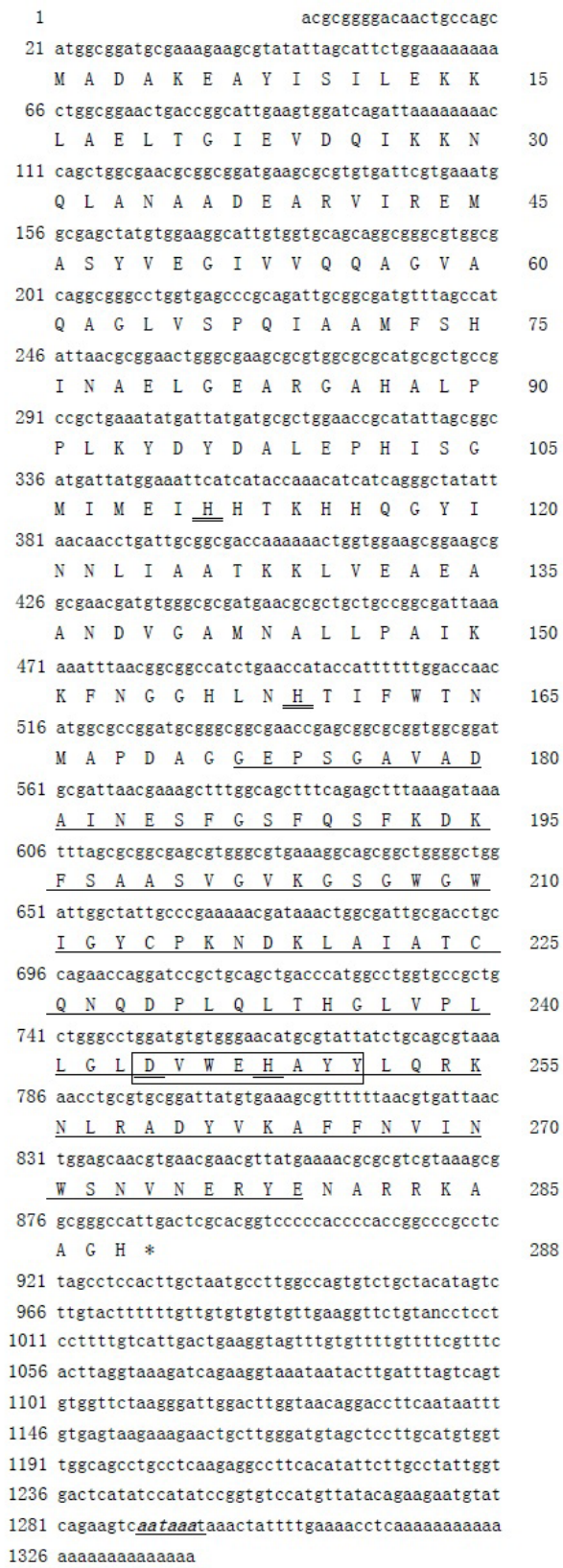

Figure 1. Complete complementary DNA (cDNA) and deduced amino acid sequences of Eriocheir sinensis cytosolic manganese superoxide dismutase (Es-cMnSOD). Nucleotides are numbered from the first base at the 5 '-end. Amino acids are numbered from the initiating methionine start codon. The general MnSOD domain is underlined. The putative MnSOD signature sequences (DVWEHAYY) are boxed. Putative manganese binding sites (i.e., H111, H159, D244, and H248) are double underlined. The asterisk $\left(^{*}\right)$ indicates the stop codon. The polyadenylation signal (aataaa) is in bold font and underlined. The full sequence was submitted to GenBank under accession No. FJ617306. 

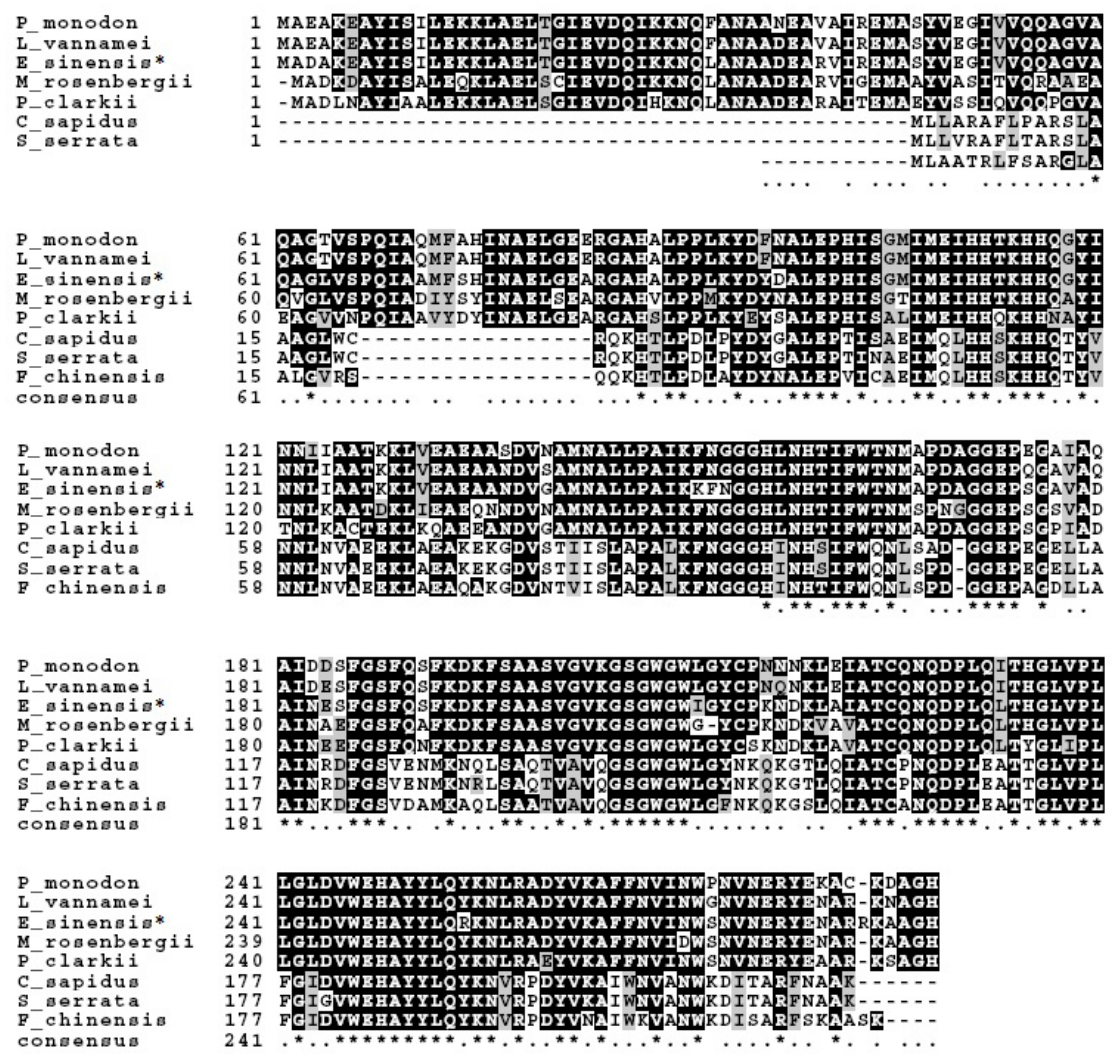

Figure 2. Alignment of Es-cMnSOD with other known species homologues, including Callinectes sapidus MnSOD (AAF74770.1), Penaeus monodon MnSOD (ACK44125.1), Macrobrachium rosenbergii MnSOD (ABU55005.1), Scylla serrata MnSOD (ACM61856.1), Litopenaeus vannamei MnSOD (ABC59529.1), Fenneropenaeus chinensis MnSOD (ABB05539.1), and Procambarus clarkii MnSOD (ABX44762.3). The shaded regions indicate the identical residues. Other conserved, but not consensus amino acids, are shaded in gray.

\section{Quantitative analysis of SOD gene expression}

Utilizing our knowledge of the activity of $\beta$-actin as an internal control in all tissues, qRT-PCR was employed to investigate the distribution of the Es-cMnSOD mRNA in different tissues. The mRNA of Es-cMnSOD was detected in all of the test tissues with different expression levels. The mRNAs of the Es-cMnSOD gene showed the highest and lowest levels of expression in the hepatopancreas and gill, respectively. Fragments were not amplified when the RNA was not reversely transcribed prior to PCR (Figure 4A).

To detect the function of SOD in the immune response in crabs, the expression of SOD in hemocytes after bacterial challenge was measured through the qRT-PCR method with $\beta$-actin as an internal control. In both the Es-cMnSOD and $\beta$-actin genes, there was only one peak at the corresponding melting temperature in the dissociation curve, indicating that the PCR was specifically amplified. Temporal expression of the Es-cMnSOD gene in the control and the bacterium-challenged tests are shown in Figure 4B. There was one peak 
in the mRNA expression profile following stimulation. Temporal expression of the Es$c M n S O D$ gene after bacterial challenge was up-regulated at $1.5 \mathrm{~h}$ after bacterial injection. Then, a step-by-step recovery at $24 \mathrm{~h}$ was conducted; at $48 \mathrm{~h}$ after bacterial injection, the expression level of the $E s-c M n S O D$ gene was similar to that observed at $1.5 \mathrm{~h}$ after bacterial injection.

Table 1. Accession number, scientific name, and source of MnSOD amino acid sequences used to construct the phylogenetic tree.

\begin{tabular}{|c|c|c|}
\hline Accession No. & Scientific name & Type of MnSOD \\
\hline DQ298208.1 & Litopenaeus vannamei & cMnSOD \\
\hline AF264030.1 & Callinectes sapidus & cMnSOD \\
\hline AF264029.1 & Callinectes sapidus & mMnSOD \\
\hline KC461130.1 & Penaeus monodon & $\mathrm{mMnSOD}$ \\
\hline JQ040506.1 & Cherax quadricarinatus & cMnSOD \\
\hline HQ852225.1 & Macrobrachium nipponense & mMnSOD \\
\hline HQ852226.1 & Macrobrachium nipponense & cMnSOD \\
\hline FM242573.1 & Carcinus maenas & $\mathrm{cMnSOD}$ \\
\hline EF427949.1 & Fenneropenaeus chinensis & $\mathrm{cMnSOD}$ \\
\hline FJ031018.1 & Portunus trituberculatus & cMnSOD \\
\hline GU213434.1 & Scylla paramamosain & cMnSOD \\
\hline FJ605170.2 & Scylla paramamosain & mMnSOD \\
\hline EU077526.1 & Macrobrachium rosenbergii & cMnSOD \\
\hline EU077525.1 & Macrobrachium rosenbergii & mMnSOD \\
\hline EU254488.3 & Procambarus clarkii & cMnSOD \\
\hline DQ205424.1 & Fenneropenaeus chinensis & mMnSOD \\
\hline GQ403706.1 & Eriocheir sinensis & $\mathrm{cMnSOD}$ \\
\hline JQ763321.1 & Cherax quadricarinatus & mMnSOD \\
\hline FJ892724.1 & Procambarus clarkii & mMnSOD \\
\hline HQ848280.1 & Tigriopus japonicus & mMnSOD \\
\hline FM213481.1 & Perisesarma bidens & mMnSOD \\
\hline FM213479.1 & Carcinus maenas & mMnSOD \\
\hline FM213477.1 & Cardisoma armatum & mMnSOD \\
\hline FM213475.1 & Segonzacia mesatlantica & mMnSOD \\
\hline FM213473.1 & Bythograea thermydron & mMnSOD \\
\hline FM213471.1 & Atelecyclus undecimdentatus & mMnSOD \\
\hline FM213469.1 & Necora puber & mMnSOD \\
\hline FM213480.1 & Cardisoma armatum & $\mathrm{mMnSOD}$ \\
\hline FM213478.1 & Perisesarma bidens & mMnSOD \\
\hline FM213476.1 & Xantho poressa & mMnSOD \\
\hline FM213474.1 & Cyanagraea praedator & mMnSOD \\
\hline FM213472.1 & Dromia personata & mMnSOD \\
\hline FM213470.1 & Cancer pagurus & mMnSOD \\
\hline AY211085.1 & Farfantepenaeus aztecus & cMnSOD \\
\hline AY211086.1 & Farfantepenaeus aztecus & mMnSOD \\
\hline AY211084.1 & Palaemonetes pugio & $\mathrm{cMnSOD}$ \\
\hline AY726542.1 & Penaeus monodon & cMnSOD \\
\hline FJ892725.1 & Uca pugnax & mMnSOD \\
\hline FM242571.1 & Cardisoma armatum & cMnSOD \\
\hline FM242569.1 & Segonzacia mesatlantica & cMnSOD \\
\hline FM242567.1 & Bythograea thermydron & cMnSOD \\
\hline FM242565.1 & Atelecyclus undecimdentatus & $\mathrm{cMnSOD}$ \\
\hline FM242563.1 & Necora puber & $\mathrm{cMnSOD}$ \\
\hline FM242572.1 & Perisesarma bidens & $\mathrm{cMnSOD}$ \\
\hline FM242570.1 & Xantho poressa & cMnSOD \\
\hline FM242568.1 & Cyanagraea praedator & cMnSOD \\
\hline FM242566.1 & Dromia personata & cMnSOD \\
\hline FM242564.1 & Cancer pagurus & cMnSOD \\
\hline DQ029053.1 & Litopenaeus vannamei & cMnSOD \\
\hline GQ181123.1 & Marsupenaeus japonicus & cMnSOD \\
\hline GQ478988.1 & Marsupenaeus japonicus & mMnSOD \\
\hline HM371157.1 & Litopenaeus vannamei & $\mathrm{Cu}-\mathrm{Zn} \mathrm{MnSOD}$ \\
\hline NM_001043619.1 & Bombyx mori & $\mathrm{Cu}-\mathrm{Zn} \mathrm{MnSOD}$ \\
\hline
\end{tabular}




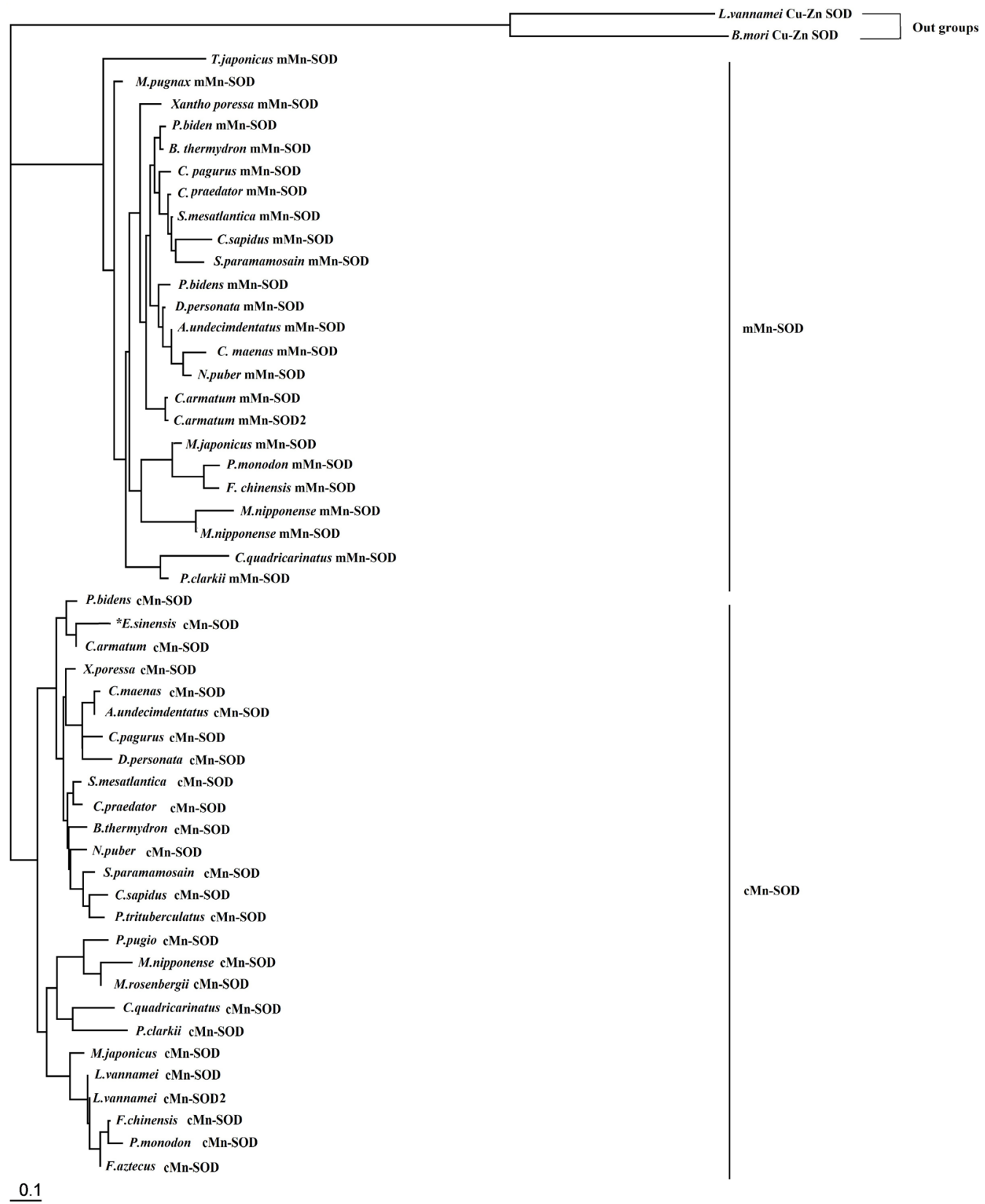

Figure 3. Neighbor-joining phylogenetic tree of Es-MnSOD from different species. The abbreviations of these proteins and their GenBank accession numbers used to construct the phylogenetic tree are given in Table 1. The Es-MnSOD is indicated with an asterisk $\left(^{*}\right)$. 
A

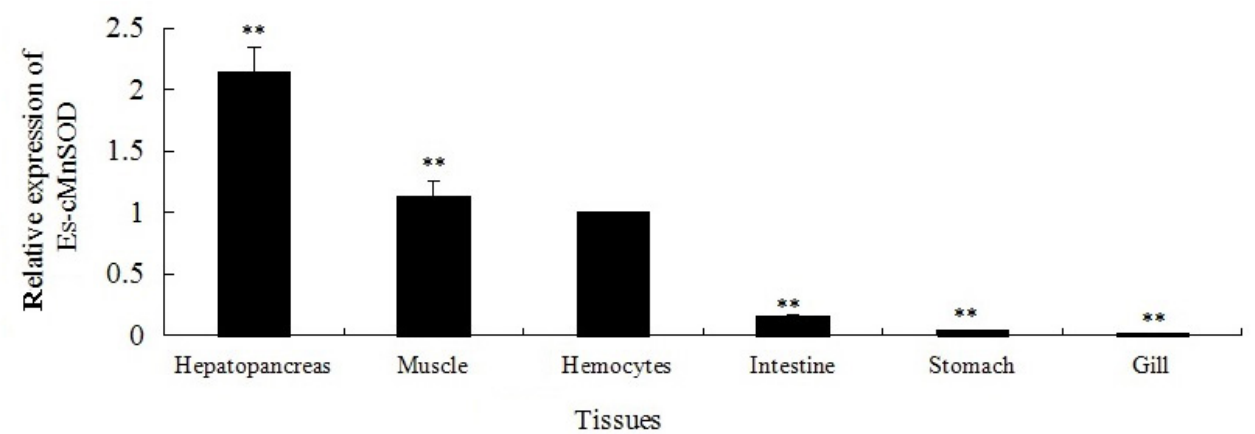

B

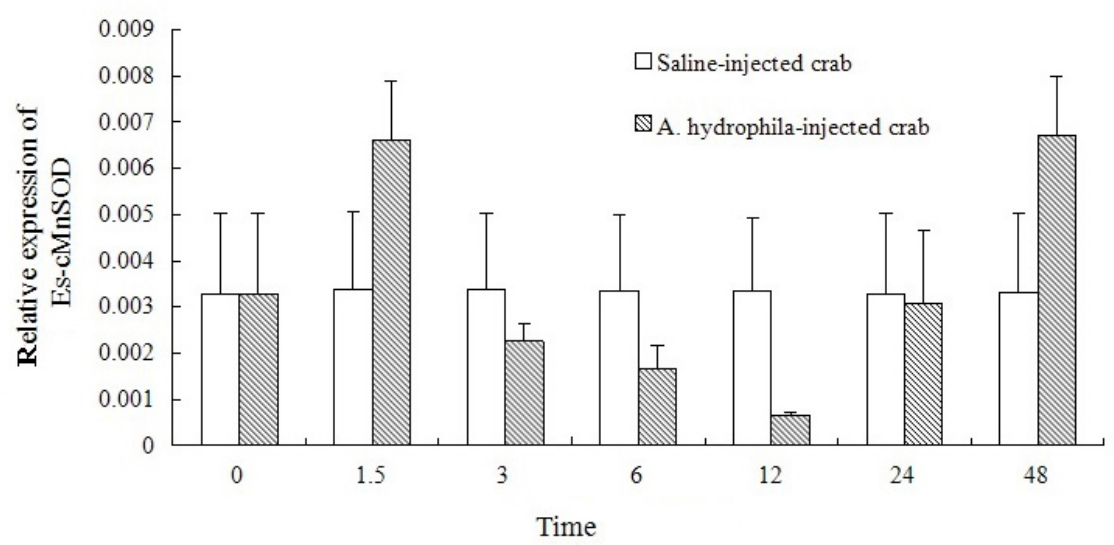

Figure 4. Expression and induction of Es-cMnSOD messenger RNA (mRNA). A. Tissue distribution of the EscMnSOD transcript measured by the SYBR-Green-based quantitative real-time reverse transcription polymerase chain reaction (qRT-PCR). The tissues, including hepatopancreas, hemocytes, gill, stomach, muscle, and intestine, were collected from the crab. All values are relative to Es-cMnSOD expression in hemocytes, which was arbitrarily set to 1 after normalization by $\beta$-actin expression. ${ }^{* *} \mathrm{P}<0.01$ for statistical significance differences of Es-cMnSOD expression when compared to that in hemocytes. B. Temporal expression profile of the Es-cMnSOD transcript in hemocytes after Aeromonas hydrophila challenge as measured by SYBR-Green-based qRT-PCR. Comparisons of the Es-cMnSOD mRNA levels (relative to $\beta$-actin mRNA) among different times were performed by the $t$-test. Values are reported as means $\pm \mathrm{SE}(\mathrm{N}=3)$.

\section{DISCUSSION}

SODs play an important role in antioxidant defense pathways, and the MnSODs comprise a major type of SOD for crustaceans. There are 2 types of MnSODs (i.e., cMnSOD and $\mathrm{mMnSOD})$. In this study, BLAST and multiple-alignment analyses demonstrated that the MnSOD isolated from E. sinensis shared high similarity with the cMnSOD in other crustacean species and was clustered into a subgroup of the cMnSODs according to the N-J-phylogenetic analysis, which indicates that the MnSOD cloned from the Chinese mitten crab is an MnSOD. We designated this gene as Es-cMnSOD. 
The full-length of Es-cMnSOD was cloned based on the EST obtained in the study. In this study, both $E s-c M n S O D$ and other arthropod $c M n S O D s$ showed several common features, including an MnSOD domain (DVWEHAYY) and 4 putative manganese binding sites $(\mathrm{H}, \mathrm{H}$, $\mathrm{D}$, and H). An arginine (R258) located at the seventh amino acids after the MnSOD domain was present. This $\mathrm{R}$ residue near the MnSOD domain was considered necessary for the structural stability of MnSOD. Among the known MnSODs, most species have an R residue in this position and only a few possess the $\mathrm{K}$ residue. The position replaced by the $\mathrm{K}$ residue reduces MnSOD activity (Gabbianelli et al., 1997). Furthermore, a polyadenylation signal (aataaa), which is known to be involved in the regulation of mRNA stability, was observed in the 3'-UTR (Malter and Hong, 1991). The conservation sequence and motif indicate the same function with that of other organisms.

In this study, the Es-cMnSOD was ubiquitous in all tissues examined, including the hepatopancreas, gill, muscle, hemocytes, stomach, and intestine. This finding is not surprising since the expression of $M n S O D$ in a wide range of cell types has been reported previously in the white shrimp L. vannamei (Gómez-Anduro et al., 2006), giant freshwater prawn M. rosenbergii (Cheng et al., 2006), red swamp crayfish P. clarkia (Zhu and Doumen, 2009) and the swimming crab Portunus trituberculatus (Li et al., 2010).

MnSOD has been considered a stress-responsive factor and its expression at the transcriptional and translational levels might be influenced by a variety of intracellular and environmental cues (Fukuhara et al., 2002). This study investigated whether acute bacterial challenge could affect the expression of cMnSOD in the Chinese mitten crab. In a bacterial challenged crab, up-regulation of Es-cMnSOD gene expression from the infected crab at 1.5 $\mathrm{h}$ post-challenge showed that the gene might be involved in temporal expression because of the invasion of heterogeneous bacteria. The significant increase in the transcript level of SOD after bacterial invasion suggests that the gene transcriptions are inducible. At $48 \mathrm{~h}$ postinjection, the transcript level in the challenged crab was higher than that in the control, suggesting that during the phagocytosis on bacteria via crab immune cells, the level of ROS increases and more SODs are needed to offset the effects of ROS. In previous study, the Chinese shrimp F. chinensis MnSOD was activated in the hemocytes and hepatopancreas when cells were infected with the white spot syndrome virus (Zhang et al., 2007), while the Crassostrea hongkongensis SOD could be induced after infection by Vibrio alginolyticus (Yu et al., 2011). Therefore, the change in transcript levels of $E s-c M n S O D$ implies that this gene may play an important role in the immune responses against infection.

In conclusion, cMnSOD cDNA was cloned for the first time from the hemocytes of E. sinensis. The sequence analysis makes it possible to visualize the specific responses of the SODs to various stress factors such as bacterial infection. The transcriptional quantification of $E s-c M n S O D$ provides valuable and reliable information for measuring oxidative-stressinduced regulation in the crab during bacterial infection. Further study is suggested to focus on the immune regulation of $E s-c M n S O D$ under different protein levels.

\section{ACKNOWLEDGMENTS}

Research supported by grants from the Special Fund for Agro-Scientific Research in the Public Interest (\#201003020, \#201203065), the National 'Twelfth Five-Year' Plan for Science \& Technology Support (\#2012BAD25B03), the National Basic Research Program 
(“973" Program, \#2014CB138603), the National Natural Science Foundation of China (\#31172422, \#31260642), the Shanghai Technology System for Chinese Mitten-Handed Crab Industry, the Natural Science Foundation of Jiangxi Province (\#20132BAB214015), the China Postdoctoral Science Foundation (\#2012M521292, \#2013T60650), the Jiangxi Postdoctoral Researcher Projects (\#2013KY23), and the Foundation of Jiangxi Educational Committee (\#GJJ12144).

\section{REFERENCES}

Arenas-Ríos E, León-Galván MA, Mercado PE, López-Wilchis R, et al. (2007). Superoxide dismutase, catalase, and glutathione peroxidase in the testis of the Mexican big-eared bat (Corynorhinus mexicanus) during its annual reproductive cycle. Comp. Biochem. Physiol. A Mol. Integr. Physiol. 148: 150-158.

Bannister JV, Bannister WH and Rotilio G (1987). Aspects of the structure, function, and applications of superoxide dismutase. CRC Crit. Rev. Biochem. 22: 111-180.

Bao Y, Li L and Zhang G (2008). The manganese superoxide dismutase gene in bay scallop Argopecten irradians: cloning, 3D modelling and mRNA expression. Fish Shellfish Immunol. 25: 425-432.

Brouwer M, Hoexum BT, Grater W and Brown-Peterson N (2003). Replacement of a cytosolic copper/zinc superoxide dismutase by a novel cytosolic manganese superoxide dismutase in crustaceans that use copper (haemocyanin) for oxygen transport. Biochem. J. 374: 219-228.

Cheng W, Tung YH, Liu CH and Chen JC (2006). Molecular cloning and characterisation of cytosolic manganese superoxide dismutase (cytMn-SOD) from the giant freshwater prawn Macrobrachium rosenbergii. Fish Shellfish Immunol. 20: 438-449.

Fridovich I (1995). Superoxide radical and superoxide dismutases. Annu. Rev. Biochem. 64: 97-112.

Fukuhara R, Tezuka T and Kageyama T (2002). Structure, molecular evolution, and gene expression of primate superoxide dismutases. Gene 296: 99-109.

Gabbianelli R, Battistoni A, Polticelli F, Meier B, et al. (1997). Effect of Lys175 mutation on structure function properties of Propionibacterium shermanii superoxide dismutase. Protein Eng. 10: 1067-1070.

Gómez-Anduro GA, Barillas-Mury CV, Peregrino-Uriarte AB, Gupta L, et al. (2006). The cytosolic manganese superoxide dismutase from the shrimp Litopenaeus vannamei: molecular cloning and expression. Dev. Comp. Immunol. 30: 893-900.

Ho YS, Howard AJ and Crapo JD (1991). Molecular structure of a functional rat gene for manganese-containing superoxide dismutase. Am. J. Respir. Cell Mol. Biol. 4: 278-286.

Johnson P (2002). Antioxidant enzyme expression in health and disease: effects of exercise and hypertension. Comp. Biochem. Physiol. C Toxicol. Pharmacol. 133: 493-505.

Jones PL, Kucera G, Gordon H and Boss JM (1995). Cloning and characterization of the murine manganous superoxide dismutase-encoding gene. Gene 153: 155-161.

Jung Y, Nowak TS, Zhang SM, Hertel LA, et al. (2005). Manganese superoxide dismutase from Biomphalaria glabrata. J. Invertebr. Pathol. 90: 59-63.

Kim KY, Lee SY, Cho YS, Bang IC, et al. (2007). Molecular characterization and mRNA expression during metal exposure and thermal stress of copper/zinc- and manganese-superoxide dismutases in disk abalone, Haliotis discus discus. Fish Shellfish Immunol. 23: 1043-1059.

Kumar S, Tamura K and Nei M (2004). MEGA3: integrated software for molecular evolutionary genetics analysis and sequence alignment. Brief Bioinform. 5: 150-163.

Lee SY and Söderhäll K (2002). Early events in crustacean innate immunity. Fish Shellfish Immunol. 12: 421-437.

Li C, He J, Su X and Li T (2011). A manganese superoxide dismutase in blood clam Tegillarca granosa: molecular cloning, tissue distribution and expression analysis. Comp. Biochem. Physiol. B Biochem. Mol. Biol. 159: 64-70.

Li JT, Chen P, Liu P, Gao BQ, et al. (2010). The cytosolic manganese superoxide dismutase cDNA in swimming crab Portunus trituberculatus: molecular cloning, characterization and expression. Aquaculture 309: 31-37.

Lin CT, Tseng WC, Hsiao NW, Chang HH, et al. (2009). Characterization, molecular modelling and developmental expression of zebrafish manganese superoxide dismutase. Fish Shellfish Immunol. 27: 318-324.

Lin YC, Lee FF, Wu CL and Chen JC (2010). Molecular cloning and characterization of a cytosolic manganese superoxide dismutase (cytMnSOD) and mitochondrial manganese superoxide dismutase (mtMnSOD) from the kuruma shrimp Marsupenaeus japonicus. Fish Shellfish Immunol. 28: 143-150.

Malter JS and Hong Y (1991). A redox switch and phosphorylation are involved in the post-translational up-regulation of the adenosine-uridine binding factor by phorbol ester and ionophore. J. Biol. Chem. 266: 3167-3171.

Genetics and Molecular Research 13 (4): 9429-9442 (2014) 
Marchand J, Leignel V, Moreau B and Chénais B (2009). Characterization and sequence analysis of manganese superoxide dismutases from Brachyura (Crustacea: Decapoda): hydrothermal Bythograeidae versus littoral crabs. Comp. Biochem. Physiol. B Biochem. Mol. Biol. 153: 191-199.

McCord JM and Fridovich I (1969). Superoxide dismutase. An enzymic function for erythrocuprein (hemocuprein). $J$. Biol. Chem. 244: 6049-6055.

Meyrick B and Magnuson MA (1994). Identification and functional characterization of the bovine manganous superoxide dismutase promoter. Am. J. Respir. Cell Mol. Biol. 10: 113-121.

Muñoz M, Cedeño R, Rodrígue J, van der Knaap WPW, et al. (2000). Measurement of reactive oxygen intermediate production in hemocytes of the penaeid shrimp, Penaeus vannamei. Aquaculture 191: 89-107.

Park SY, Kim YS, Yang DJ and Yoo MA (2004). Transcriptional regulation of the Drosophila catalase gene by the DRE/ DREF system. Nucleic Acids Res. 32: 1318-1324.

Smith VJ, Brown JH and Hauton C (2003). Immunostimulation in crustaceans: does it really protect against infection? Fish Shellfish Immunol. 15: 71-90.

St Clair DK, Oberley TD, Muse KE and St Clair WH (1994). Expression of manganese superoxide dismutase promotes cellular differentiation. Free Radic. Biol. Med. 16: 275-282.

Trachootham D, Lu W, Ogasawara MA, Nilsa RD, et al. (2008). Redox regulation of cell survival. Antioxid. Redox Signal. 10: $1343-1374$.

Vaughan M (1997). Oxidative modification of macromolecules minireview series. J. Biol. Chem. 18513.

Wan XS, Devalaraja MN and St Clair DK (1994). Molecular structure and organization of the human manganese superoxide dismutase gene. DNA Cell Biol. 13: 1127-1136.

Wang W and Gu Z (2002). Rickettsia-like organism associated with tremor disease and mortality of the Chinese mitten crab Eriocheir sinensis. Dis. Aquat. Organ. 48: 149-153.

Wang W, Zhu N, Gu Z, Du K, et al. (2002). Study on the transmission of tremor disease (TD) in the Chinese mitten crab, Eriocheir sinensis (Crustacea: Decapoda). J. Invertebr. Pathol. 81: 202-204.

Weisiger RA and Fridovich I (1973). Superoxide dismutase. Organelle specificity. J. Biol. Chem. 248: 3582-3592.

Xu HS, Shu MA, Zhan XA and Wang SX (2002). Identification of Vibrio parahemolyticus isolated from cultured Eriocheir sinensis and pathogenicity of its extracellular products. J. Fish. China 26: 357-362.

Yu Z, He X, Fu D and Zhang Y (2011). Two superoxide dismutase (SOD) with different subcellular localizations involved in innate immunity in Crassostrea hongkongensis. Fish Shellfish Immunol. 31: 533-539.

Zhang Q, Li F, Wang B, Zhang J, et al. (2007). The mitochondrial manganese superoxide dismutase gene in Chinese shrimp Fenneropenaeus chinensis: cloning, distribution and expression. Dev. Comp. Immunol. 31: 429-440.

Zhang ZW, Li Z, Liang HW, Li L, et al. (2011). Molecular cloning and differential expression patterns of copper/zinc superoxide dismutase and manganese superoxide dismutase in Hypophthalmichthys molitrix. Fish Shellfish Immunol. 30: 473-479.

Zhao D, Song SH, Wang Q and Zhang X (2009). Discovery of immune-related genes in Chinese mitten crab (Eriocheir sinensis) by expressed sequence tag analysis of hemocytes. Aquaculture 287: 297-303.

Zhu H and Doumen C (2009). Identification of a cytoplasmic manganese superoxide dismutase (cMnSOD) in the red swamp crawfish, Procambarus clarkii: cDNA cloning and tissue expression. Zool. Sci. 26: 284-288. 